\title{
Caution before fractionating COVID-19 vaccines
}

To the Editor-We agree with Cowling et al. ${ }^{1}$ that dose fractionation to make more effective use of limited supplies of vaccine antigen to achieve higher vaccination coverage rates and thus prevent more deaths during the COVID-19 pandemic must be seriously considered. However, such a strategy will, importantly, need supportive evidence, and there are additional considerations that require deliberation and scrutiny.

As Cowling et al. stated, the World Health Organization (WHO) has indeed endorsed dose-sparing vaccine strategies due to limited vaccine supply ${ }^{1}$. Examples of this include vaccines against yellow fever and polio. However, the rationale for fractionating vaccines against yellow fever in emergency situations was that this live vaccine has excess potency per dose, far above the minimum threshold required by the $\mathrm{WHO}^{2,3}$. Thus, a one-fifth dose is generally still higher than the minimum threshold of antigen needed. Another strategy for dose sparing is intradermal application. Intradermal application allows the use of lower doses and has been endorsed by the WHO for vaccines against rabies and polio ${ }^{4,5}$. Although intradermal administration may enable a reduction in dose volume, it may also change the immunogenicity and safety and increase the reactogenicity profile of vaccines. Indeed, increased reactogenicity of intradermal application was observed for mRNA vaccines against influenza in a phase 1 trial $^{6}$. Scaling up intradermal administration at a global level would also have major programmatic implications that would require substantial investments in training and logistics and the supply of special syringes and would initially delay rather than accelerate vaccine rollout.

The potential for dose reduction will depend on the platform technology (e.g., mRNA, vectored, protein, subunit or inactivated vaccine). Reducing the dose by one half, one third or one fifth could theoretically be considered with various options, such as fractionated doses for the priming schedule, or fractionated doses for any booster doses, should booster doses prove to be needed in the future.

However, policy recommendations for reducing doses should be made only after an extensive evidence review of immunogenicity and safety. Emergency-use listing for all vaccines against COVID-19 for which the WHO has issued policy recommendations has been based on the evidence derived from phase 3 trials using the dose that was identified in phase 1 dose-finding studies. Additional clinical studies would be needed to inform policy on the use of doses lower than those determined from dose-finding efforts, given that an exact immune correlate for protection has not yet been obtained for most vaccines against COVID-19. Additionally, there is no correlate for duration of protection, although in general, higher antibody levels are thought to be correlated with better efficacy and duration.

For the phase 3 trial of the ChAdOx1 vaccine, one study arm received half of the currently recommended dose. An initial half dose elicited a lower immune response than a full dose did, while a half dose followed by full dose elicited immune responses after the second dose similar to those elicited by two full doses. However, immune responses were lower after two half doses, and were also lower after a full dose followed by a half dose, than after two full doses ${ }^{7}$. The clinical relevance of these findings remain uncertain.

The mRNA-1273 vaccine is currently undergoing studies of a half dose versus a full dose for booster doses, and data are awaited. A phase 2 trial for the mRNA1273 vaccine compared $50 \mu \mathrm{g}$ versus 100 $\mu \mathrm{g}$ as the primary series and found that seroconversion rates were lower after the first vaccination with $50 \mu \mathrm{g}$ as the first dose than after first vaccination with 100 $\mu \mathrm{g}$, but they were similarly high after two vaccinations with both doses, although geometric mean titers were generally higher for the $100-\mu \mathrm{g}$ dosing regimen than for the $50-\mu \mathrm{g}$ dosing regimen ${ }^{8}$. Although the initial efficacy findings for the mRNA-1273 vaccine at a dose of $100 \mu \mathrm{g}$ were equivalent to the efficacy results for the BNT162b2 mRNA vaccine at a dose of $30 \mu \mathrm{g}$, the durability of high neutralizing-antibody titers has now been shown to be higher for the mRNA-1273 vaccine than for the BNT162b2 mRNA vaccine?.

The protection against breakthrough infections by the Delta and Beta variants of SARS-CoV-2 was also shown to be higher with the mRNA-1273 vaccine than with the BNT162b2 mRNA vaccine ${ }^{10}$. Whether this was due to the higher dosing is uncertain; further evaluation of mechanisms underlying differences in effectiveness, such as dosing regimens and vaccine composition, is needed.

We are not aware of studies using reduced doses for the other vaccines against COVID-19 that have received WHO emergency-use listing, such as the inactivated whole-virus vaccines from Sinovac and Sinopharm, the Pfizer-BioNTech BNT162b2 vaccine, and the Janssen Ad26.COV2.S vaccine.

Several questions are relevant for informing policy recommendations on fractionation of vaccines against COVID-19. Do fractionated doses result in non-inferior neutralizing-antibody levels, cell-mediated immunity, cross-protection against variants and duration of immune response? What is the clinical protection against various clinical endpoints (death, severe disease, and mild-to-moderate or asymptomatic infection) and against different variants of concern? Are fractionated doses equally effective in certain subpopulations, in particular in immunocompromised people, those with comorbidities or those with immunosenescence related to older age? How does the safety profile of fractionated doses compare with that of full-dose schedules?

There are also several programmatic considerations, as currently licensed vaccine formulations may not be suitable for the administration of fractionated doses. Small dose volumes may require special syringes, and for certain multi-dose vaccines, there may be difficulties in adjusting the diluent volume. Other programmatic issues may limit feasibility and hamper rapid rollout.

Proposing the use of fractionated doses also needs to be considered in the context of community acceptance, given that the rapid rollout of vaccines against COVID-19 has already led to confusion and conspiracy theories; fractionated doses may further exacerbate vaccine hesitancy. Furthermore, if fractionated doses result in lower neutralizing-antibody levels, there may be a need for more booster doses, which would make the vaccine rollout programmatically and financially more challenging.

Although we acknowledge the potential public-health benefits and potential cost reduction of dose-sparing strategies to increase vaccine supply and accelerate population-level vaccination coverage, there is currently insufficient supportive evidence to recommend the use of fractionated doses. Additionally, programmatic and operational implications of this strategy may hamper rapid implementation efforts.

Any use of a fractionated dose at this point in time constitutes off-label use. We encourage more research in the area, with a particular emphasis on research into using fractionated doses in children 
and adolescents, in whom lower antigen content may elicit immune responses similar to those elicited by full doses in adults. Proactive research into decreasing antigen for booster doses is of particular interest, should booster doses be required in the future. Vaccine effectiveness and safety studies will be needed as part of the follow-up.

Annelies Wilder-Smith",2,3凶, Shalini Desai', Alejandro Cravioto ${ }^{4}$, Hanna Nohynek ${ }^{5}$ and Joachim Hombach?

${ }^{I}$ Immunization, Vaccines and Biologicals, World Health Organization, Geneva, Switzerland. ${ }^{2} I n s t i t u t e$ of Social and Preventive Medicine, University of Berne, Berne, Switzerland. ${ }^{3}$ Heidelberg Institute of Global Health, University of Heidelberg,
Heidelberg, Germany. ${ }^{4}$ Faculty of Medicine, National Autonomous University of Mexico, Mexico City, Mexico. ${ }^{5}$ Department of Health Security at the Finnish Institute for Health and Welfare,

Helsinki, Finland.

$凶_{e \text {-mail:wildersmitha@who.int }}$

Published online: 22 September 2021

https://doi.org/10.1038/s41591-021-01534-z

\section{References}

1. Cowling, B. J., Lim, W. W. \& Cobey, S. Nat. Med. 27, 1321-1323 (2021)

2. Roukens, A. H. E. \& Visser, L. G. J. Travel Med. 26, taz024 (2019)

3. World Health Organization. Wkly. Epidemiol. Rec. 25, 345-356 (2017).

. Okayasu, H. et al. J. Infect. Dis. 216, S161-S167 (2017).

5. World Health Organization. Wkly. Epidemiol. Rec. 16, 201-220 (2018)

6. Feldman, R. A. et al. Vaccine 37, 3326-3334 (2019).
7. Barrett, J. R. et al. Nat. Med. 27, 279-288 (2021).

8. Chu, L. et al. Vaccine 39, 2791-2799 (2021).

9. Abe, K. T. et al. Preprint at medRxiv https://doi.org/10.1101/ 2021.08.06.21261721 (2021).

10. Puranik, A. et al. Preprint at medRxiv https://doi.org/10.1101/ 2021.08.06.21261707 (2021).

Acknowledgements

A.W.-S. is a consultant to the WHO; S.D. and J.H. are on the staff of the WHO; and A.C. and H.N. are unpaid advisers to the WHO and are members of the WHO's Strategic Advisory Group of Experts on vaccination. The authors alone are responsible for the views expressed and they do not necessarily represent the decisions, policies or views of the World Health Organization.

Author contributions

A.W.-S. wrote the first draft, and all authors contributed to the final manuscript.

Competing interests

The authors declare no competing interests. 